Instructions for authors, subscriptions and further details:

\title{
La Dependencia Emocional y su Relación con el Sexismo en Adolescentes
}

Miguel Cañete Lairla ${ }^{1}$

Luis Domingo Díaz Sánchez ${ }^{2}$

1) University of Zaragoza, Spain

2) University of Extremadura, Spain

Date of publication: October $25^{\text {th }}, 2019$

Edition period: October 2019-February 2020

To cite this article: Cañete Lairla, M. \& Díaz Sánchez, L.D. (2019). La Dependencia Emocional y su Relación con el Sexismo en Adolescentes, International Journal of Sociology of Education, 8(3), 236-260. doi: 10.17583/rise.2019.4198

To link this article: http://dx.doi.org/10.17583/rise.2019.4198

PLEASE SCROLL DOWN FOR ARTICLE

The terms and conditions of use are related to the Open Journal System and to Creative Commons Attribution License (CC-BY) 


\section{Emotional Dependence and its Relationship with Sexism in Adolescents}

Miguel Cañete Lairla

University of Zaragoza
Luis Domingo Díaz Sánchez

University of Extremadura

(Received: 21 March 2019; Accepted: 17 August 2019; Published: 25 October 2019)

\section{Abstract}

Adolescence is a stage of crisis in which the values and important people of childhood to leave one step to others to form the basis of adult setting up. Among the variables that will become protagonists of this transition, this study will translate into the important role of emotional dependence and its relationship with ambivalent sexism in the function of sex and age, as well as studying other psychosocial variables that are involved in adolescents' behavior. From a sample of 85 Secondary Education students, the data is analyzed using correlation tests, media comparison and variable control tests. The results show the differential evolution of dependence based on sex and age, the relationship with sexism, family and social isolation, guilt, self-esteem and submission.

Keywords: adolescents, emotional dependence, ambivalent sexism, psychosocial variables 


\section{La Dependencia Emocional y su Relación con el Sexismo en Adolescentes}

Miguel Cañete Lairla

University of Zaragoza
Luis Domingo Díaz Sánchez

University of Extremadura

(Recibido: 21 Marzo 2019; Aceptado: 17 Agosto 2019; Publicado: 25 Octubre 2019)

\section{Resumen}

La adolescencia es una etapa de crisis en la que los valores y las personas importantes de la infancia empiezan a dejar paso a aquellos otros que constituirán la base de la formación del adulto. De entre las variables que serán protagonistas de esta transición, este estudio pretende mostrar el importante papel de la dependencia emocional y su relación con el sexismo ambivalente en función del sexo y la edad, así como estudiar otras variables psicosociales que están implicadas en la conducta de los adolescentes. A partir de una muestra de 85 estudiantes de Educación Secundaria y Bachiller, se analizan los datos obtenidos en las escalas aplicadas utilizando pruebas de correlación, comparación de medias y de control de variables. Los resultados muestran la evolución diferencial de la dependencia en función del sexo y la edad, su relación con el sexismo, el aislamiento familiar y social, la culpa, la autoestima y la sumisión.

Palabras clave: adolescentes, dependencia emocional, sexismo ambivalente, variables psicosociales. 


\section{Cañete \& Díaz-Adolescencia y dependencia emocional}

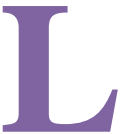

a violencia entre iguales es un problema con una presencia importante en las aulas de secundaria en la mayor parte de los países del mundo (Juvonen y Graham, 2014). Su presencia en los centros educativos, donde los adolescentes pasan una gran parte de las horas del día, interfiere notablemente en el proceso de enseñanza/aprendizaje, así como en su desarrollo evolutivo, con consecuencias siempre negativas para su bienestar, salud psicológica y relaciones sociales (Estévez y Jiménez, 2015).

Especialmente importante es la violencia ejercida por motivos sexistas. Ligada a esta idea, podemos definir el sexismo como una ideología de género. Algunos autores como Pratto y Walker (2004), consideran que este tipo de ideología tiende a sustentar el desequilibrio de poder existente entre hombres y mujeres en la sociedad en la que vivimos. Por un lado, el sexismo caracteriza a los hombres como más fuertes, autónomos y dominantes que las mujeres (Expósito y Herrera, 2009; Glick y Whitehead, 2010), capacitándoles para realizar otros tipos de trabajos y definiéndoles como "más capaces" que las mujeres. Sin embargo, estas últimas deben preocuparse de las labores de crianza y cuidado del hogar para ser consideradas "buenas madres y esposas". Una visión dotada de una connotación machista.

Todas estas desigualdades de poder, generan un contexto propicio para la justificación de la dominación de los hombres sobre las mujeres, convirtiéndolas en más vulnerables a medida que va pasando el tiempo. La culpa atribuida a mujeres víctimas de violencia merece atención, ya que es un aspecto particular de la justificación de la violencia de género, que como hemos mencionado anteriormente, es respaldada por el sexismo (Lee, Fiske, Glick y Chen, 2010). Por tanto, se trata de un problema que continúa requiriendo de una especial atención por parte de los profesionales y de una comprensión profunda por parte de los investigadores. Un mundo en el que aún queda mucho que decir.

El objetivo de este estudio consiste en analizar la dependencia emocional en jóvenes adolescentes, así como las diferencias por sexo y edad en dependencia emocional y evaluar la relación de dicha dependencia con otras variables psicosociales. Además de la autoestima, frecuentemente ligada a la dependencia, se analizarán variables como el aislamiento, la culpa, la empatía y la sumisión, además de su relación con el sexismo tanto hostil 
como benévolo.

Las actuaciones preventivas en violencia de género han de centrarse en los sistemas de creencias presentes en niños y adolescentes para neutralizar en lo posible los sesgos de corte sexista y los estereotipos alimentados socioculturalmente (Recio, Cuadrado y Ramos, 2007). Asimismo, variables asociadas con la violencia en parejas de adultos han de considerarse también como elementos importantes que se van gestando a lo largo del desarrollo de la persona y que facilitan la aparición y consolidación del fenómeno de la violencia de género.

En primer lugar, la dependencia emocional se define como un patrón persistente de necesidades emocionales que no están satisfechas y que se intentan cubrir a través otras personas de una forma desadaptativa (Castelló, 2000; Rodríguez de Medina, 2013). En las relaciones de pareja alude a un tipo de relación interpersonal establecida entre una persona y su pareja caracterizada por la necesidad extrema de afecto, una necesidad excesiva de obtener la aprobación de los demás, lo que permitiría sentirnos mejor con nosotros mismos; pudiendo evitar el sentimiento de soledad (Castelló, 2005; Urbiola, 2014). De ahí su relación con la autoestima.

Otros autores como Skvortsova y Shumskiy (2014), señalan que las personas dependientes sienten la imposibilidad de imaginarse su existencia sin la pareja. La persona que muestra una dependencia emocional puede establecer relaciones en las que existe una gran necesidad afectiva que le lleva a establecer relaciones conocidas como asimétricas; adoptando una posición de subordinación o sumisión en relación con su pareja, y llevando a cabo una gran cantidad de actuaciones con el objetivo de evitar que la relación concluya (Izquierdo y Gómez-Acosta, 2013).

Respecto a los factores que inciden en la ocurrencia e intensidad de esta característica de la personalidad son, como cabía esperar, muy variados. En primer lugar, deberíamos destacar los procesos que conducen a la autonomía e independencia del niño desde su más tierna infancia y entender el fenómeno de la dependencia como un malfuncionamiento de estos procesos que conduce a la inestabilidad emocional. La importancia del tipo de apego y otras experiencias tempranas marcan las preferencias por un tipo $\mathrm{u}$ otro de relación emocional con las personas (López Sánchez, 2008; Chopik, Edelstein y Fraley, 2013). En los adolescentes podemos distinguir tres etapas al respecto de la adquisición de la independencia: la primera de recelo y 
confrontación que se produce entre los 12 y 14 años y que incluye un vacío emocional; en la segunda, estos conflictos llegan a su apogeo entre los $15 \mathrm{y}$ 17 años dando como resultado una mayor independencia, integración y madurez; en la tercera se produciría una vuelta a los afectos y valores provenientes de la familia y tendría lugar entre los 18 y los 21 años (Iglesias Diz, 2013).

En este movimiento de ida y vuelta van a cobrar gran importancia las variables de personalidad asociadas a cada persona y las experiencias anteriores a la etapa adolescente. Así, la crianza con carencias afectivas en la etapa infantil podría conducir a los sujetos a buscar la sensación de protección y seguridad que tanto desean, estableciendo relaciones de dependencia (Rusbya, Harrisb y Taskera, 2013). Esta situación conduciría a una incapacidad para poder disfrutar de relaciones interpersonales de un modo saludable y satisfactorio, impidiéndoles conseguir tanto un bienestar psíquico como un equilibrio personal y social (Ara, 2012). De la misma manera, se pueden generar a su vez, relaciones de total sumisión hacia la otra persona, anulándose como sujetos, dado que su motivación más importante sería la de sentirse acompañados, independientemente de la calidad de la relación (Janin, 2011). Es decir, se generan en la persona dudas sobre su valía y el desarrollo de sus capacidades; estando sometidos a la constante aprobación de otras personas. Esto último, acarrearía que la persona quede anulada en todas sus facultades, convirtiéndose en una figura de compañía de aquella que la maneja y manipula a su interés. Por esta razón, resulta tan necesaria una buena autoestima, que nos permita visualizar con claridad cuáles son nuestras fortalezas y debilidades, para poder establecer una serie de metas ajustadas tanto a nuestras características personales como a nuestros intereses (De la Villa y Sirvent, 2009).

Para la persona dependiente emocionalmente, el trauma que supone la ruptura es verdaderamente devastador, ya que constituye con frecuencia la aparición de síntomas depresivos tales como: sentimientos de rechazo, negación y abandono que pueden desembocar en suicidios consumados (Lemos, Londoño y Zapata, 2007). También, pueden padecer síntomas relacionados con la ansiedad, sentimientos de desvalimiento y vacío emocional, junto con una constante insatisfacción, que anula sus facultades cuando intenta realizar alguna actuación que se propone (Hirigoyen, 2013; Morgan y Clark, 2010). El aislamiento o falta de apoyo tanto familiar como 
social serían variables relevantes.

Ligado a lo expuesto hasta el momento, podemos afirmar que la dependencia emocional también ha sido estudiada en relación con el género. Charkow y Nelson (2000), señalaron que las mujeres han sido educadas en la creencia de que la felicidad consiste en tener una pareja sentimental (Sánchez-Aragón, 2009) lo que conlleva todo un conjunto de mitos acerca del amor romántico (Bosch et al., 2007). Este tipo de educación favorece la idealización del amor y de las relaciones de pareja (González-Ortega, Echeburúa y de Corral, 2008), lo que podría contribuir a la dependencia emocional en las mujeres. Sin embargo, otros estudios han mostrado que los varones adolescentes obtienen puntuaciones más altas en dependencia emocional (Urbiola y Estévez, 2015).

El desarrollo del adolescente es diferente en función del sexo y, por supuesto, la edad de la persona. También en la infancia encontramos características diferenciales en el desarrollo evolutivo de niños y niñas en aspectos como habilidades lingüísticas, espaciales o matemáticas (López Sánchez, 2008), aunque este dimorfismo se inicia ya durante el desarrollo embrionario e implica diferencias en la formación no solamente de la estructura anatómica sino también de la cerebral (Saro, 2009; Moreira-Filho et al., 2018). Estas cuestiones son importantes a la hora de hablar del desarrollo humano, especialmente en las etapas infantil y adolescente haciendo necesario que las investigaciones incluyan las variables sexo y edad con el objetivo de contrastar las evidencias que existen hasta el momento en muchas variables que son especialmente dependientes de la etapa evolutiva.

Además de las diferencias físicas obvias, el desarrollo psicosocial comprende cuatro aspectos fundamentales como son: la dependenciaindependencia, la preocupación por el aspecto corporal, la integración en el grupo de amigos y el desarrollo de la identidad (Iglesias Diz, 2013). La adquisición de estos aspectos es diferente para cada persona aunque pueden encontrarse unas pautas comunes en todos los adolescentes pero que van a estar también influidas por el sexo y la edad. Las relaciones sentimentales que establecen los jóvenes sirven como oportunidades para ampliar experiencias e intereses; y así, poder enriquecer la identidad de la persona (Sánchez et al., 2011). La función de estas primeras relaciones de pareja consistiría en encontrar quiénes son, cómo de atractivos resultan, aprender a 


\section{Cañete \& Díaz-Adolescencia y dependencia emocional}

interactuar en una relación de pareja y ganar estatus en su grupo de pares (Domínguez, 2008). Sin embargo, Castelló (2000, 2005) señala que la dependencia emocional puede darse igualmente sin que sea necesario tener una pareja.

La dependencia emocional se ha asociado frecuentemente con sintomatología depresiva y ansiedad entre otras manifestaciones psicopatológicas (Rodríguez et al., 2010). Los resultados reflejan una relación positiva entre las puntuaciones de dependencia emocional y la sintomatología ansiosa depresiva, indicando que a mayor puntuación en dependencia emocional, mayor sintomatología disfuncional. Existía una relación proporcionalmente directa. Estos resultados son acordes a los propuestos por autores como Greenberg y Bornstein (1988), que señalan que las puntuaciones en depresión podrían ser explicadas por las puntuaciones obtenidas en dependencia. En este sentido, Huprich (2003), señaló que un exceso de dependencia podría ser un punto determinante en el desarrollo de un estado depresivo, ya que el relato de los pacientes se compone en la mayoría de los casos, por una sensación elevada de soledad. Sentimiento de aislamiento. Dichos resultados se ven apoyados también en estudios realizados previamente en los que se han investigado los aspectos adaptativos y desadaptativos de este fenómeno, en los cuales se ha encontrado evidencia de la influencia de la dependencia emocional tanto en trastornos psicológicos como en trastornos orgánicos (Bornstein, 1994). Todo esto nos da una idea de la importancia de esta variable en la salud de las personas.

Estos resultados también coinciden con los estudios llevados a cabo por otros autores, en los que se afirma, además, que a mayor puntuación en dependencia emocional, menor será la autoestima de estos jóvenes. Es decir, cuanto más baja sea la autoestima, más intensa será la necesidad de buscar apoyo en el otro (Wei, Vogel, Ku y Zakalik, 2005). Por tanto, los resultados encontrados en este estudio refuerzan la literatura científica previa, que hace referencia a la relación entre la dependencia emocional y otras características psicológicas.

Además, se ha analizado si la variable dependencia emocional es predictora de la sintomatología ansiosa y depresiva, y de la autoestima; encontrándose que la subescala de evitar estar solo fue predictora de la sintomatología ansiosa, mientras que en el caso de la autoestima fueron 
predictoras tanto de la necesidad de agradar como de evitar estar solo. Esta última hace referencia a las acciones que lleva a cabo la persona dependiente para no estar sola, ya que necesita sentirse querida, siendo esta su motivación para involucrarse en las relaciones sentimentales (Urbiola, Estévez e Iraurgi, 2014).

Respecto a las variables psicosociales utilizadas en este estudio, escogeremos aquellas que se han demostrado importantes en su relación con la violencia verbal o psicológica en parejas de adultos (Cañete-Lairla y GilLacruz, 2017). Además de la autoestima, claramente relacionada con la dependencia, se analizarán otras variables como el aislamiento (tanto familiar como social), la culpa, la empatía y la tendencia a la sumisión. Pasamos a describir brevemente estas variables.

\section{Autoestima}

Se refiere a la valoración afectiva y subjetiva que la persona hace de su autoconcepto. Una baja autoestima en la víctima se encuentra relacionada con sentimientos de dependencia, así como con una mayor intensidad y duración de los procesos de violencia recibida por parte de su pareja (Jordan, Campbell y Follingstad, 2010). Se encuentra relacionada, como hemos visto, con otras manifestaciones psicopatológicas y suele ser atraer el foco de intervención en víctimas de violencia (Labrador, Fernández-Velasco y Rincón, 2006; Mecca, Smelser y Vasconcellos, 1989), además de considerarse un factor protector importante frente a estresores percibidos (Cabanach, Souto Gestal, Freire Rodríguez y Ferradás Canedo, 2015).

\section{Aislamiento}

Puede ser entendida como la ausencia de contacto significativo y satisfactorio con otras personas con diversas consecuencias para la salud y las relaciones violentas (Hendy, Eggen, Gustitus, McLeod y Ng, 2003). Si bien algunos autores consideran esta variable a nivel global, es importante diferenciar entre el aislamiento respecto a los miembros de la propia familia (tanto nuclear como ampliada) y el aislamiento social respecto de amistades, compañeros de clase, vecinos, etc (Cañete-Lairla y Gil-Lacruz, 2017; Echeburúa, Amor y de Corral, 2002; Wright, 2015). 


\section{Culpa}

La tendencia a atribuir la causa de los sucesos negativos a la propia responsabilidad o acciones está estrechamente relacionada con el locus de control interno (Rotter, 1964). Las personas con un locus de control interno tienen sentimientos de culpa y vergüenza de mayor intensidad que aquellas con un locus de control externo (Palomar y Valdés, 2004). Esta culpabilidad o exceso de responsabilidad puede contribuir a aceptar o soportar un trato negativo por parte de otras personas, especialmente la pareja.

\section{Empatía}

Se entiende como una identificación con los pensamientos, sentimientos y afectos de otra persona (Davis, 1983). Un exceso de empatía puede estar asociado a una mayor tolerancia y aceptación a la hora de sufrir manipulaciones de los sentimientos por parte de otra persona (Rabelo y Ronaldo Pilati, 2017). Además, también se han encontrado puntuaciones bajas en empatía en agresores de violencia machista (Echeburúa, Sarasua, Zubizarreta y de Corral, 2009; Boira y Esteban, 2010; Loinaz, Echeburúa y Ullate, 2012)

\section{Sumisión}

Es la tendencia a ser excesivamente obsequioso y servil (Patró, Corbalán y Limiñana, 2007) o a necesitar la aprobación de los demás para las decisiones y conductas propias. Implica un sacrificio de los propios intereses y necesidades para satisfacer las de los demás formando parte del llamado síndrome de la mujer maltratada (Walker, 2012)

\section{Sexismo}

La clave de la existencia de formas de sexismo nuevas y tradicionales, reside en la teoría de sexismo ambivalente (Glick y Fiske, 2001), según la cual, el sexismo tiene un componente hostil y otro benévolo. El primero, hace referencia al sexismo tradicional, centrado en creencias sobre la inferioridad de las mujeres frente a los hombres. Éstas, como resultado, no valoran sus 
capacidades y sienten inseguridad ante determinadas situaciones lo que dificulta alcanzar los objetivos que se propongan a lo largo de su vida.

Sin embargo, la discriminación abierta y hostil es ilegal en los países occidentales por lo que cada día cobra más fuerza otro tipo de sexismo más encubierto y sutil, que expresa el deseo de los hombres por cuidar a las mujeres, protegerlas y adorarlas, y por resaltar las habilidades de la mujer en las tareas a las cuales les relega el estereotipo de rol de género tradicional femenino (Velasco, 2009). Es el sexismo benévolo una visión de protección más que de posesión, con un tono afectivo positivo y se trata, en cualquier caso, de una visión limitada de la mujer fomentando un tratamiento desigual y perjudicial hacia las mujeres (Garaigordobil y Aliri, 2011; Recio et al., 2007).

\section{Objetivos}

La comprensión del fenómeno de la violencia requiere la consideración de muchas y diversas variables tanto de la personalidad como del entorno en el que el individuo desarrolla su vida cotidiana, así como de la etapa evolutiva. Estas variables interactúan para generar diferentes respuestas ante las demandas de la situación y de la relación con los otros, produciendo a veces respuestas que suponen una agresión a las personas que nos rodean, ya sea verbal, física o psicológica. Resulta muy fácil condenar la violencia pero lo verdaderamente difícil es entenderla. Es por esto que si no llegamos a comprender los mecanismos que subyacen a la violencia difícilmente podremos intervenir para evitarla. Con esta investigación se pretende arrojar un poco más de luz sobre este fenómeno en los adolescentes y en especial a través del estudio de la personalidad dependiente.

De esta manera se plantean como objetivos los siguientes:

- Conocer el desarrollo de la dependencia en adolescentes entre $15 \mathrm{y}$ 18 años.

- Establecer el grado de asociación entre la dependencia emocional y el nivel de sexismo tanto benévolo como hostil.

- Estudiar las relaciones de la dependencia con otras variables psicosociales que son importantes en los estudios de víctimas de violencia en la pareja.

- Determinar la influencia de la edad y el sexo en las variables 
significativas.

\section{Método}

\section{Participantes}

La muestra la componen 85 participantes de un Instituto de Educación Secundaria de la provincia de Cáceres, 35 de $4^{\circ}$ de Educación Secundaria Obligatoria (ESO) y 50 de $2^{\circ}$ de Bachillerato, sin que existan diferencias significativas en las variables relevantes excepto en edad, obviamente, y en dependencia como se estudiará posteriormente. En la muestra se encuentran 39 mujeres y 46 varones, con una proporción de varones significativamente más alta en $4^{\circ}$ de $\operatorname{ESO}(29,4 \%)$ y mayor proporción de mujeres en $2^{\circ}$ de Bachiller (34,1\%). El estadístico $\chi^{2}$ de homogeneidad arrojó un valor de 7,18 con un nivel crítico $p=, 014$. Ante la falta de homogeneidad en la variable curso, descartaremos dicha variable para tener en cuenta únicamente la edad, tanto como variable cuantitativa como agrupada en cuatro categorías según los valores de 15 años, 16, 17 y 18 o más.

\section{Instrumentos}

Escala de Detección de Sexismo en Adolescentes (DSA). Compuesta por 26 ítems, 16 diseñados para la medida del sexismo hostil y 10 para la evaluación del sexismo benévolo. La escala de respuesta a los ítems es tipo Likert con 6 anclajes (desde $1=$ "totalmente en desacuerdo" hasta $6=$ "totalmente de acuerdo"). Según sus autores, el ajuste al modelo bifactorial propuesto fue bastante aceptable, con valores superiores a ,90 para todos los índices de bondad de ajuste del análisis factorial confirmatorio (Recio et al., 2007). El estudio de la consistencia interna y la validez convergente presentan valores en línea con los obtenidos en otras escalas de sexismo ambivalente como el Ambivalent Sexism Inventory (ASI) según estos mismos autores.

Escala de variables psicosociales asociadas con violencia machista. Compuesta por 23 ítems, 4 diseñados para medir la dependencia, 4 para el déficit de autoestima, 4 para el aislamiento ( 2 para aislamiento familiar y 2 para aislamiento social), 2 para culpa, 4 para empatía y 5 para sumisión. El 
análisis factorial confirmatorio del estudio original arroja índices de ajuste muy adecuados, con una alta consistencia interna (Cañete, 2014). Los valores de respuesta para cada uno de los ítems de las escalas son 0,1 y 2 . Estas variables están altamente relacionadas con la violencia verbal en parejas adultas (Cañete-Lairla y Gil-Lacruz, 2017)

Variables sociodemográficas. Curso, edad, sexo y nacionalidad.

\section{Procedimiento}

Para llevar a cabo la recogida de datos, se contactó con el equipo directivo del centro exponiéndole los contenidos y finalidad de la investigación. Dada la minoría de edad de los participantes se solicitó la autorización de sus padres, así como el propio consentimiento de los alumnos. El estudio tuvo lugar en el periodo escolar, concretamente en las horas de tutoría, lejos de los exámenes finales y de la prueba de acceso a la universidad, dado que un par de grupos pertenecían a $2^{\circ}$ de Bachillerato. Antes de administrar los cuestionarios a los estudiantes, se les explicaron también a ellos los objetivos del estudio y se incidió en que la participación era voluntaria y que los datos serían tratados de manera confidencial y anónima. El investigador estuvo presente en la toma de datos para explicar los instrumentos empleados y resolver cualquier duda que pudiera surgir.

Para los análisis estadísticos se ha utilizado el programa IBM SPSS (Statistical Package for the Social Science) versión 24.

\section{Resultados}

Como se ha comentado, el desarrollo evolutivo de los adolescentes depende de muchas variables y se lleva a cabo de forma diferente en varones y en mujeres. Por lo tanto, es muy posible que los resultados encontrados vayan a estar en relación con el sexo y la edad, entre otras variables, obligándonos a un análisis de las variables en cruzamiento con estas dos. Es por esto que habrá que tener cautela a la hora de generalizar los resultados y explorar las posibles opciones alternativas que puedan llevarnos a la comprensión del desarrollo adolescente.

Vemos en primer lugar los estadísticos descriptivos para las variables estudiadas y las diferencias en función del sexo (Tabla 1). 
Tabla 1.

Estadísticos descriptivos y diferencias por sexo

\begin{tabular}{|c|c|c|c|c|c|}
\hline & \multicolumn{2}{|c|}{ Mujer $(n=39)$} & \multicolumn{2}{|c|}{ Varón $(n=46)$} & \multirow{2}{*}{ t de Student } \\
\hline & Media & SD & Media & SD & \\
\hline Edad & 17,00 & 1,26 & 16,57 & 1,26 & N.S. \\
\hline Sexismo global & 1,53 & 0,48 & 1,94 & 0,74 & $-2,95 * *$ \\
\hline Sexismo Benévolo & 2,11 & 0,88 & 2,29 & 0,94 & N.S. \\
\hline Sexismo Hostil & 1,17 & 0,32 & 1,71 & 0,74 & $-4,26 * * *$ \\
\hline Aislamiento Familiar & 0,19 & 0,39 & 0,26 & 0,42 & N.S. \\
\hline Aislamiento Social & 0,41 & 0,46 & 0,38 & 0,37 & N.S. \\
\hline Déficit de Autoestima & 0,79 & 0,47 & 0,60 & 0,39 & $2,12 *$ \\
\hline Culpa & 0,85 & 0,38 & 0,71 & 0,39 & N.S. \\
\hline Dependencia & 0,60 & 0,40 & 0,77 & 0,43 & N.S. \\
\hline Empatía & 1,41 & 0,34 & 1,45 & 0,32 & N.S. \\
\hline Sumisión & 1,10 & 0,25 & 1,31 & 0,27 & $-3,75^{* * *}$ \\
\hline
\end{tabular}

N.S.: No significativo; $* * \mathrm{p}<, 01 ; * * * \mathrm{p}<, 001$

No existen diferencias significativas en función de la nacionalidad y dado el escaso número de sujetos con valores diferentes a la española $(n=3)$ no se tendrá en cuenta esta variable en los análisis.

El estudio global exploratorio de la variable dependencia proporciona relaciones significativas con cuatro de las variables estudiadas: edad ( $\mathrm{r}=-$ ,222 y $\mathrm{p}=, 042)$, sexismo hostil $(\mathrm{r}=, 216$ y $\mathrm{p}=, 047)$, aislamiento familiar $(\mathrm{r}$ $=, 255$ y $\mathrm{p}=, 018)$ y culpa $(\mathrm{r}=, 262$ y $\mathrm{p}=, 016)$. Esto nos indica una relación inversa con la variable edad, mostrando que la dependencia emocional iría disminuyendo conforme aumenta la edad. El resto de correlaciones son positivas indicando una relación directa: a mayor dependencia mayor sexismo hostil, aislamiento familiar y culpa. Sin que podamos indicar hasta ahora las variables antecedentes y consecuentes.

Dependencia y edad

El sexo es una variable determinante tanto en el desarrollo físico como psicosocial de tal forma que estamos obligados a diferenciar los resultados 
en función de esta variable para poder averiguar en qué variables influye y como permite al adolescente ir configurando su identidad.

El estudio de la dependencia en función de la edad nos indica que dicha relación es únicamente significativa en el grupo de mujeres adolescentes $(r=$ $-, 432$ y $\mathrm{p}=, 006)$ siendo una variable irrelevante en el caso de los varones $(\mathrm{r}$ $=-, 007$ y $\mathrm{p}>, 05)$.

El estudio de estas variables mediante el ANOVA confirma los hallazgos con un valor del estadístico para el grupo de mujeres de $\mathrm{F}=3,38(\mathrm{p}=, 029)$ y $\mathrm{F}=0,43(\mathrm{p}>, 05)$ para el grupo de varones. Mientras que en el grupo de mujeres la dependencia disminuye considerablemente de los 15 a los 16 años para mantenerse en niveles bajos en los dos años siguientes, en el grupo de varones asciende hasta tomar su valor máximo a los 17 años, desde donde comienza su descenso como indica la figura 1.

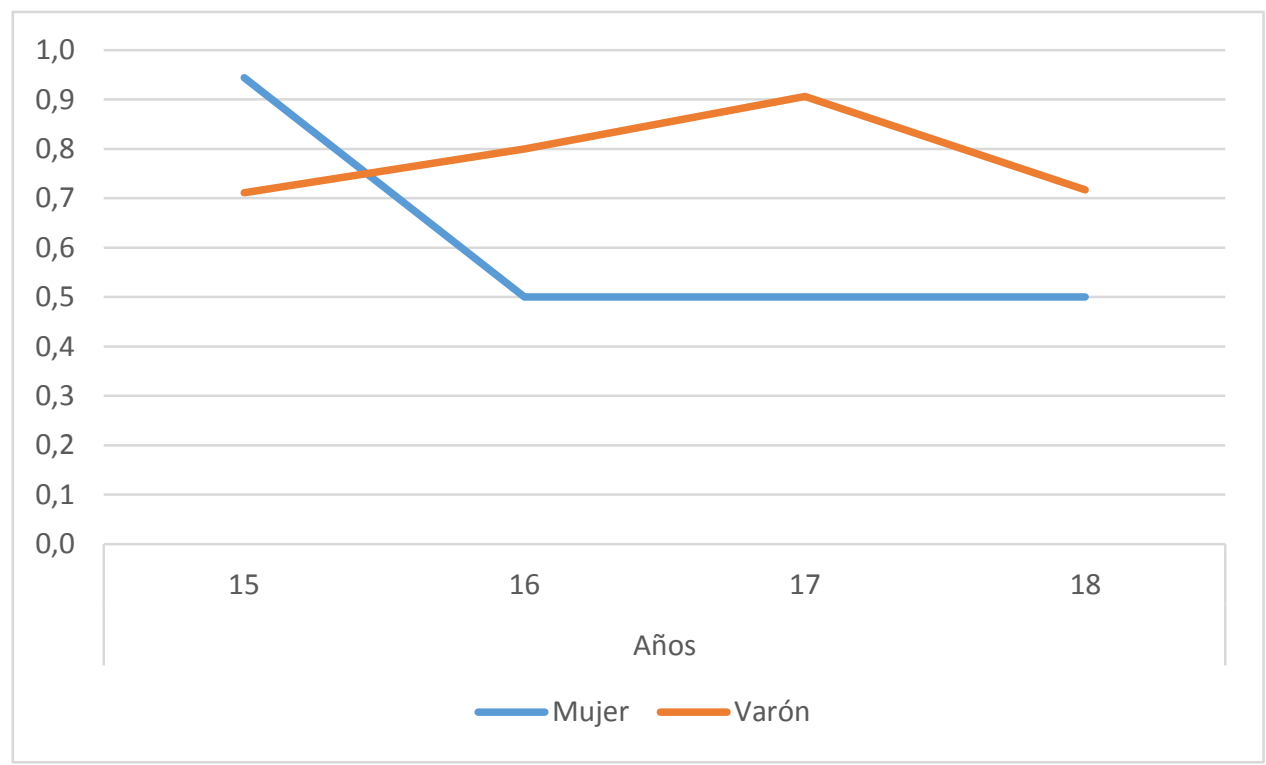

Figura 1. Evolución de la dependencia en función de la edad 


\section{Dependencia y Sexismo Hostil}

En primer lugar, podemos decir que el sexismo hostil tiene una carga afectiva negativa, en el que se muestra una visión inferior de la mujer frente al hombre, que es quien domina la situación. Las actitudes sexistas no permiten considerar a la mujer en situación de igualdad, aun desempeñando una responsabilidad laboral muy similar (Walton y Politano, 2014).

Como se ha indicado, existe una relación entre dependencia y sexismo hostil. En el estudio por sexos no se aprecian diferencias significativas, debido probablemente a los pequeños tamaños muestrales de los grupos desglosados. Sin embargo, en la literatura revisada esta última variable tiene una mayor presencia entre los varones (Díaz Sánchez, 2016; Recio et al., 2007).

Puesto que la evolución de la dependencia está relacionada con la edad, como hemos visto, hemos de controlar esta variable para poder atribuir las variaciones en sexismo hostil a la dependencia emocional. Realizado un análisis de covarianza (ANCOVA) entre la dependencia y el sexismo hostil, y tomando como covariable la edad, los resultados nos permiten afirmar que existen diferencias significativas en sexismo hostil en función de la dependencia después de haber eliminado el posible efecto de la edad ( $\mathrm{F}=$ $3,84, \mathrm{p}=, 001$ у $\left.\eta^{2}=0,29\right)$.

\section{Dependencia y Aislamiento Familiar}

El aislamiento familiar se entiende como una falta de apoyo proveniente de la familia: padres, hermanos. Es una variable que está presente en muchos estudios en las víctimas de violencia y probablemente relacionada con el estilo de apego. Una falta de apego seguro se traduce en una menor autonomía por parte del niño en relación a la exploración e interacción con el entorno, incluidas las personas más cercanas. La crisis de la adolescencia también plantea un apartamiento de la confianza y el afecto de los padres, para dirigirlo a los iguales, que al parecer se manifiesta como aislamiento familiar.

El análisis más profundo de las correlaciones nos descubre que la relación entre dependencia y aislamiento familiar se produce únicamente en los varones $(\mathrm{r}=, 303 \mathrm{y} \mathrm{p}=, 041)$ no siendo significativa en el grupo de 
mujeres.

Hay que mencionar que la correlación con la variable aislamiento social es negativa, aunque no es significativa en ninguno de los grupos. Los niveles de edad tampoco establecen diferencias significativas en esta variable.

\section{Dependencia y Culpa}

La culpa o responsabilidad excesiva es también, como ya hemos indicado, una variable que nos encontramos presente en mujeres víctimas de violencia machista.

El estudio de la relación encontrada en la muestra global se debe a la relación existente entre estas variables en el grupo de mujeres $(r=, 492$ y $\mathrm{p}=$ ,001) no existiendo relación para el grupo de varones.

\section{Dependencia y Autoestima}

No encontramos relación entre la dependencia y la autoestima. Sin embargo, esta variable indica un mayor déficit de autoestima en mujeres $(M=0,79)$ que en varones $(\mathrm{M}=0,60), \mathrm{t}_{83}=2,12 \mathrm{y} \mathrm{p}=, 037$, y está relacionada con el aislamiento familiar también en el grupo de mujeres, $\mathrm{r}=, 33 \mathrm{y} \mathrm{p}=, 043$.

No existe relación entre dependencia y sumisión, aunque se han encontrado fuertes diferencias en función del sexo, con valores más altos de sumisión en varones que en mujeres $(\mathrm{t}=-3,75 \mathrm{y} \mathrm{p}<, 001)$

\section{Discusión y Conclusiones}

En este estudio se ha examinado la relación de la dependencia emocional en los adolescentes con algunas variables psicosociales, así como con el sexismo ambivalente, entendido éste como precursor de conductas relacionadas con la violencia en la pareja. Las variables sexo y edad tienen una gran importancia como variables de control, lo que se manifiesta en los resultados obtenidos.

Los hallazgos muestran las diferencias existentes en sexismo hostil en función del sexo con mayores puntuaciones en el grupo de varones, lo que se traduce en puntuaciones también superiores en sexismo global. Sin embargo, estas diferencias se dan únicamente en los grupos de edad de 17 y 18 años, 


\section{Cañete \& Díaz-Adolescencia y dependencia emocional}

que es cuando la dependencia toma los valores más altos en el grupo de varones. El control de la variable edad hace plausible que dicho sexismo aparezca como una reacción a las necesidades de dependencia en los varones adolescentes de estas edades. Estos resultados concuerdan parcialmente con otras investigaciones ya que, si bien el sexismo hostil presenta habitualmente valores mayores en el grupo de varones adolescentes (Recio et al., 2007), las puntuaciones en dependencia emocional no arrojan valores significativamente diferentes entre estos grupos (Pradas y Perles, 2012). Sin embargo, estudios que utilizan el control de la variable edad sí que indican mayores puntuaciones en dependencia emocional en varones, con diferencias en función de la edad (Urbiola y Estévez, 2015).

Destacan asimismo las diferencias encontradas en autoestima con un mayor déficit en esta variable en el grupo de mujeres y sin que se encuentren diferencias por grupos de edad, ni por niveles de dependencia, aunque existen estudios que asocian el déficit de autoestima como variable predictora de una mayor dependencia (Urbiola, Estévez, Iruarrizaga y Jauregui, 2017). Posiblemente sean los roles de género asignados tradicionalmente a las mujeres los que estén relacionados con estas diferencias, ya que, el proceso de socialización que comienza a desarrollarse en estas edades impone una mayor restricción de posibilidades y experiencias para las adolescentes o también por la autoevaluación de las mujeres adolescentes basada en la aprobación de los otros significativos más que en sus propios logros y habilidades (Naranjo y González, 2012).

Parece ser que la falta de apoyo familiar estaría estrechamente relacionada con la dependencia y la necesidad de buscar relaciones sociales sustitutorias entre los iguales. Se trataría, en parte, de los conflictos que se inician en la adolescencia temprana (Iglesias Diz, 2013), encaminados a desprenderse del control parental que se había ido aceptando desde la infancia y que ahora tenderían a dirigir las necesidades de relación hacia los iguales en mayor medida. Por otra parte, la falta de apoyo familiar aparece también asociada al déficit de autoestima que se presenta en las adolescentes, confirmando la necesidad de aprobación como la contribución más importante en el grupo de mujeres.

Por último, existe una fuerte relación entre culpa y dependencia en el grupo de mujeres adolescentes, en donde la culpa aparecería asociada al vacío emocional, miedo al abandono y otros sentimientos negativos. 
Mientras los varones tenderían más a la búsqueda de sensaciones nuevas las mujeres emplearían más mecanismos de negación y falta de afrontamiento con el consiguiente sentimiento de culpa (Moral y Sirvent, 2009).

Durante la infancia, niños y niñas dependen en gran medida de las normas, valores y afectos de sus padres. Es en la adolescencia cuando los chavales empiezan a buscar su autonomía en todos los aspectos, algo que se produce gradualmente. Al igual que en otras variables, el desarrollo de la mujer precede en el tiempo al del hombre en lo que se refiere a la adquisición de la independencia y la autonomía personales. Mientras en el varón, la dependencia alcanza su máximo sobre los 17 años para empezar a descender a partir de aquí, en la mujer los valores de dependencia son ya muy bajos a los 16 años, manteniéndose estables en los años siguientes.

Estos estudios nos dan idea, por un lado, de la complejidad de las relaciones entre las variables que inciden sobre la personalidad de los adolescentes y por otro, de la importancia de la dependencia emocional como precursora de ciertas conductas y actitudes asociadas con el sexismo.

Sería recomendable continuar esta línea de investigación ampliándola a lo largo de toda la adolescencia utilizando mayores tamaños muestrales. Asimismo, sería conveniente diferenciar entre la autoestima proveniente de los padres y la de los iguales (Naranjo y González, 2012), ya que la separación respecto de los padres es imprescindible en esta etapa para que el adolescente pueda alcanzar la autonomía e independencia deseada. Esa separación ha de ir acompañada de una aproximación a los iguales, que puede o no tener éxito, pero que debe ser suficientemente satisfactoria para no dañar la autoestima global del adolescente.

Se ha podido ver en este estudio la importancia de controlar los posibles efectos de la edad y del sexo. Si bien podemos entender la adolescencia como un proceso global y generalizado de tránsito desde la infancia a la adultez, no todas las personas hacen este recorrido de la misma manera ni al mismo tiempo.

Finalmente, además de la obtención de datos de los estudiantes a través de los cuestionarios, sería conveniente contar con el testimonio de alumnos, padres y profesores, con el objetivo de enriquecer las investigaciones llevadas a cabo hasta el momento. La prevención se convierte en un pilar fundamental para llevar a cabo programas psicoeducativos que nos permitan el diagnóstico y la posterior intervención sobre las actitudes de los 
adolescentes en aquellos contextos en los que el individuo actúa. Además, el conocimiento y la comprensión tanto de los procesos como de las variables implicadas resultan fundamentales en la prevención y en la formación del profesorado a la hora de implementar estrategias de actuación más efectivas.

\section{Referencias}

Ara, C. (2012). El vínculo de apego y sus consecuencias para el psiquismo humano. Papeles de Psicoanálisis, 32, 54-62.

Boira, S., \& Esteban, P. J. (2010). Psicopatología, características de la violencia y abandonos en programas para hombres violentos con la pareja: resultados en un dispositivo de intervención. Psicothema, 22(4), 593-599.

Bornstein, R. F. (1994). Adaptative and maladaptative aspects of dependency: An integrative review. American Journal of Orthopsychiatry, 64, 622-635.

Bosch, E., Ferrer, M., García, E., Ramis, M. C., Mas, M. C., Navarro, C., \& Torrens, G. (2007). Del mito del amor romántico a la violencia contra las mujeres en la pareja. Madrid: Instituto de La Mujer, Ministerio de Igualdad.

Cabanach, R. G., Souto Gestal, A., Freire Rodríguez, C., \& Ferradás Canedo, M. D. M. (2015). Relaciones entre autoestima y estresores percibidos en estudiantes universitarios. European Journal of Education and Psychology, 7(1), 43. doi: 10.30552/ejep.v7i1.100

Cañete, M. (2014). Variables psicosociales en mujeres víctimas de violencia machista. Tesis doctoral. Universidad de Zaragoza, Zaragoza.

Cañete-Lairla, M., \& Gil-Lacruz, M. (2017). Psychosocial Variables Associated With Verbal Abuse as a Form of Intimate Partner Violence Against Women in a Spanish Sample. Journal of Aggression, Maltreatment \& Trauma, 27(3), 1-19. doi:

10.1080/10926771.2017.1320343

Castelló, J. (2000). Análisis del concepto "dependencia emocional". I Congreso Virtual de Psiquiatría 1 de Febrero-15 de Marzo 2000. Conferencia 6-CI-A. Recuperado de http://www.psiquiatria.com/congreso/mesas/mesa6/conferencias/6_ci_ a.htm

Castelló, J. (2005). Dependencia emocional. Características y tratamiento. 


\section{Madrid: Alianza Editorial.}

Charkow, W. B. y Nelson, E. S. (2000). Relationship dependency, dating violence and scripts of female. Journal of College Counselling, 3, 1217.

Chopik, W. J., Edelstein, R. S., \& Fraley, R. C. (2013). From the Cradle to the Grave: Age Differences in Attachment From Early Adulthood to Old Age: Attachment From Early to Older Adulthood. Journal of Personality, 81(2), 171-183. doi: 10.1111/j.1467-6494.2012.00793.x

Davis, M. H. (1983). Measuring individual differences in empathy: Evidence for a multidimensional approach. Journal of Personality and Social Psychology, 44(1), 113-126.

De la Villa, M. y Sirvent, C. (2009). Dependencia afectiva y género: perfil sintomático diferencial en dependientes afectivos españoles.

Interamerican Journal of Psychology, 43, 230-240.

Díaz Sánchez, L. D. (2016). El sexismo ambivalente y su relación con la autoestima en educación secundaria y bachiller. Trabajo de fin de Máster. Universidad de Zaragoza.

Domínguez, L. (2008). La adolescencia y la juventud como etapas del desarrollo de la personalidad. Distintas concepciones en torno a la determinación de sus límites y regularidades. Boletín Electrónico de Investigación de la Asociación Oaxaqueña de Psicología, 4, 69-76.

Echeburúa, E., Amor, P. J., \& De Corral, P. (2002). Mujeres maltratadas en convivencia prolongada con el agresor: variables relevantes.

Recuperado de http://e-

spacio.uned.es:8080/fedora/get/bibliuned:AccionPsicologica2002numero2-0003/Documento.pdf

Echeburúa, E., Sarasua, B., Zubizarreta, I., \& Corral, P. de. (2009).

Evaluación de la eficacia de un tratamiento cognitivo-conductual para hombres violentos contra la pareja en un marco comunitario: una experiencia de 10 años (1997-2007). International Journal of Clinical and Health Psychology, 9(2), 199-217.

Estévez, E., \& Jiménez, T. I. (2015). Conducta agresiva y ajuste personal y escolar en una muestra de estudiantes adolescentes españoles. Universitas Psychologica, 14(1), 111-124.

Expósito, F. \& Herrera, M. C. (2009). Social perception of violence against women: Individual and psychosocial characteristics of victims and 
abusers. The European Journal of Psychology Applied to the Legal Context, 1, 123-145.

Garaigordobil, M., \& Aliri, J. (2011). Sexismo hostil y benevolente: relaciones con el autoconcepto, el racismo y la sensibilidad intercultural. Journal of Psychodidactics, 16(2). Recuperado de http://www.ehu.eus/ojs/index.php/psicodidactica/article/view/998

Glick, P., \& Fiske, S. T. (2001). Ambivalent sexism. Advances in Experimental Social Psychology, 33, 115-188.

Glick, P. \& Whitehead, J. (2010). Hostility toward men and the perceived stability of male dominance. Social Psychology, 41, 177-185.

González-Ortega, I., Echeburúa, E. y de Corral, P. (2008). Variables significativas en las relaciones violentas en parejas jóvenes: una revisión. Psicología Conductual, 16, 207-225.

Greenberg, R. P. y Bornstein, R. F. (1988). The dependent personality: II.

Risk for psychological disorders. Journal of Personality Disorders, 2, 136-143.

Hendy, H., Eggen, D., Gustitus, C., McLeod, K., \& Ng, P. (2003). Decision to leave scale: Perceived reasons to stay in or leave violent relationships. Psychology of Women Quarterly, 27(2), 162-173.

Hirigoyen, M. F. (2013). El acoso moral. El maltrato psicológico de la vida cotidiana (25.a ed.). Barcelona: Paidós.

Huprich, S. K. (2003). Depressive personality and its relationship to depressed mood, interpersonal loss, negative parental perceptions, and perfectionism. The Journal of Nervous and Mental Disease, 191, 7379.

Iglesias Diz, J. L. (2013). Desarrollo del adolescente: aspectos físicos, psicológicos y sociales. Pediatría integral, XVII(2), 88-93.

Izquierdo, S. A. y Gómez-Acosta, A. (2013). Dependencia afectiva: abordaje desde una perspectiva contextual. Psychologia. Avances de la disciplina, 7, 81-91.

Janin, B. (2011). Los trastornos tempranos en la estructuración del psiquismo: la historia. Buenos Aires: Editorial Noveduc.

Jordan, C. E., Campbell, R., \& Follingstad, D. (2010). Violence and Women's Mental Health: The Impact of Physical, Sexual, and Psychological Aggression. Annual Review of Clinical Psychology, 6(1), 607-628. doi: 10.1146/annurev-clinpsy-090209-151437 
Juvonen, J, \& Grahamn S. (2014). Bullying in schools: the power of bullies and the plight of victims. Annual Review of Psychology, 65, 159-185. Labrador, F., Fernández-Velasco, R., \& Rincón, P. (2006). Eficacia de un programa de intervención individual y breve para el trastorno por estrés postraumático en mujeres víctimas de violencia doméstica. International Journal of Clinical and Health Psychology, 6(3), 527547.

Lee, T., Fiske, S., Glick, P., \& Chen, Z. (2010). Ambivalent sexism in close relationships: (Hostile) power and (benevolent) romance shape relationship ideals. Sex Roles, 62, 583-601. doi: 10.1007/s11199-0109770-x

Lemos, M., Londoño, N. y Zapata, J. (2007). Distorsiones cognitivas en personas con dependencia emocional. Informes Psicológicos, 9, 5569.

Loinaz, I., Echeburúa, E., \& Ullate, M. (2012). Estilo de apego, empatía y autoestima en agresores de pareja. Terapia psicológica, 30(2), 61-70. López Sánchez, F. (2008). Necesidades en la infancia y en la adolescencia. Respuesta familiar, escolar y social. Madrid: Pirámide.

Mecca, A. M., Smelser, N. J., \& Vasconcellos, J. (1989). The Social importance of self-esteem. Berkeley: University of California Press.

Moral, M. V., \& Sirvent, C. (2009). Dependencia Afectiva y Género: Perfil Sintomático Diferencial en Dependientes Afectivos Españoles. Revista interamericana de psicología, 43(2), 230-240.

Moreira-Filho, C. A., Bando, S. Y., Bertonha, F. B., Ferreira, L. R., Vinhas, C. de F., Oliveira, L. H. B., ... Carneiro-Sampaio, M. (2018). Minipuberty and Sexual Dimorphism in the Infant Human Thymus. Scientific Reports, 8(1). doi: 10.1038/s41598-018-31583-3

Morgan, T. A. y Clark, L. A. (2010). Passive-submissive and activeemotional trait dependency: Evidence for a two-factor model. Journal of Personality, 78, 1325-1352.

Naranjo, C. R., \& González, A. C. (2012). Autoestima en la adolescencia: análisis y estrategias de intervención. International Journal of Psychology, 15.

Palomar, J., \& Valdés, L. M. (2004). Pobreza y locus de control. Revista interamericana de psicología, 38(2), 225-240.

Patró, R., Corbalán, F. J., \& Limiñana, R. M. (2007). Depresión en mujeres 
maltratadas: relaciones con estilos de personalidad, variables contextuales y de la situación de violencia. Anales de psicología, 23(1), 118-124.

Pradas, E., \& Perles, F. (2012). Resolución de conflictos de pareja en adolescentes, sexismo y dependencia emocional. Quaderns de Psicología, 14(1), 16.

Pratto, F. \& Walker, A. (2004). The basis of gendered power. In A.H. Eagly, A. E. Beall, \& R. J. Sternberg (Eds.), The psychology of gender (2nd ed., pp. 242-268). New York: Guilford.

Rabelo, A., \& Ronaldo Pilati, R. P. (2017). Empathy is a stable predictor of compassionate emotions independent of an attribution of responsibility manipulation. Universitas Psychologica, 16(1). doi: 10.11144/Javeriana.upsy16-1.espc

Recio, P., Cuadrado, I., \& Ramos, E. (2007). Propiedades psicométricas de la Escala de Detección de Sexismo en Adolescentes (DSA). Psicothema, 19(3), 522-528.

Rodríguez de Medina, I. (2013). La dependencia emocional en las relaciones interpersonales. ReiDoCrea. Revista electrónica de investigación Docencia Creativa, 2, 143-148.

Rodríguez, L., López-Cepero, J., Rodríguez, F. J., Bringas, C., Antuna, M. A. y Estrada, C. (2010). Validación del Cuestionario de Violencia entre Novios (CUVINO) en jóvenes hispanohablantes: análisis de resultados en España, México y Argentina. Anuario de Psicología Clínica y de la Salud, 6, 45-52.

Rodríguez-Castro, Y., Lameiras-Fernández, M., Carrera-Fernández, M.-V., \& Vallejo-Medina, P. (2013). La fiabilidad y validez de la escala de mitos hacia el amor: las creencias de los y las adolescentes. Revista de Psicología Social, 28(2), 157-168. doi:

10.1174/021347413806196708

Rotter, J. B. (1964). Psicología clínica. México: UTEHA. Rusbya, J., Harrisb, J. M. y Taskera, F. (2013). Female interpersonal dependency: genetic and environmental components and its relationship to depression as a function of age. Aging \& Mental Health, 17, 1044-1051.

Sánchez-Aragón, R. (2009). Expectativas, percepción de estabilidad y estrategias de mantenimiento en las relaciones amorosas. Enseñanza e 
Investigación en Psicología, 14, 229-243.

Sánchez, L., Gutiérrez, M., Herrero, N., Ballesteros, M., Izzedin, R. y

Gómez, A. (2011). Representaciones sociales del noviazgo, en adolescentes escolarizados de estratos bajo, medio y alto, en Bogotá.

Revista de Salud Pública, 13, 79-88.

Saro, I. (2009). Transexualidad: una perspectiva transdisciplinaria. México

D.F.: Editorial Alfil. Recuperado de

http://public.eblib.com/choice/publicfullrecord.aspx?p=3206044

Skvortsova, S. N. y Shumskiy, V. B. (2014). Existential-phenomenological analysis of dependence in close interpersonal relationships.

Existenzanalyse, 31, 4-13.

Urbiola, I. (2014). Violencia recibida, ejercida y percibida en las relaciones de noviazgo de jóvenes y adolescentes. Universidad de Deusto.

Bilbao. Tesis doctoral.

Urbiola, I., Estévez, A. y Iraurgi, I. (2014). Dependencia emocional en el noviazgo de jóvenes y adolescentes (DEN): desarrollo y validación de un instrumento. Ansiedad y Estrés, 20, 101-114.

Urbiola, I. y Estévez, A. (2015). Dependencia emocional y su relación con estructuras inadaptadas tempranas en las relaciones de noviazgo en jóvenes y adolescentes. Psicología Conductual, 23, 571-587.

Urbiola, I., Estévez, A., Iruarrizaga, I., \& Jauregui, P. (2017). Dependencia emocional en jóvenes: relación con la sintomatología ansiosa y depresiva, autoestima y diferencias de género. Ansiedad y Estrés, 23(1), 6-11. doi: 10.1016/j.anyes.2016.11.003

Valle, L., \& de la Villa Moral, M. (2018). Emotional dependence and adult attachment style in dating relationships in spanish young people. Revista Iberoamericana De Psicologia y Salud, 9(1), 27-41. doi: 10.23923/rips.2018.01.013

Velasco, S. (2009). Sexos, género y salud. Teoría y métodos para la práctica clínica y programas de salud. Madrid: Minerva.

Walker, L. E. (2012). El síndrome de la mujer maltratada. Bilbao: Desclée de Brouwer.

Walton, R. O., \& Politano, P. M. (2014). Gender-Related Perceptions and Stress, Anxiety, and Depression on the Flight Deck. Aviation Psychology and Applied Human Factors, 4(2), 67-73. doi: 10.1027/2192-0923/a000058 
260 Cañete \& Díaz-Adolescencia y dependencia emocional

Wei, M., Vogel, D. L., Ku, T. Y. y Zakalik, R. A. (2005). Adult attachment, affect regulation, negative mood, and interpersonal problems: The mediating roles of emotional reactivity and emotional cutoff. Journal of Counseling Psychology, 52, 14.

Wright, E. M. (2015). The Relationship Between Social Support and Intimate Partner Violence in Neighborhood Context. Crime \& Delinquency, 61(10), 1333-1359. doi: 10.1177/0011128712466890

Miguel Cañete Lairla is professor at the University of Zaragoza, Spain

Luis Domingo Díaz Sánchez is professor at the University of Extremadura, Spain

Contact Address: mcanete@unizar.es 